\title{
Increased frequency of integrons and $\beta$-lactamase-coding genes among extraintestinal Escherichia coli isolated with a 7-year interval
}

\author{
Joanna Mokracka • Anna Oszyńska • \\ Adam Kaznowski
}

Received: 18 April 2012/Accepted: 10 August 2012/Published online: 4 September 2012

(C) The Author(s) 2012. This article is published with open access at Springerlink.com

\begin{abstract}
We analyzed the level of antimicrobial resistance, and the presence of integrons and $\beta$ lactamase-coding genes in 69 clinically relevant Escherichia coli strains originating from extraintestinal infections isolated in 1999-2001 and 2008-2010. Comparison of the two groups showed significant differences in drug resistance frequency, and the presence of integron and $\beta$-lactamase-coding genes. The frequency of resistance to all antimicrobials beside imipenem, streptomycin, piperacillin/tazobactam, and sulfamethoxazole increased significantly, especially towards aminoglycosides, $\beta$-lactams and fluoroquinolones. Similarly, we noticed an increase in the number of strains with integrons from 31.6 to $80.7 \%$. The presence of integrase genes was associated with elevated frequency of resistance to each antimicrobial tested besides imipenem, piperacillin/tazobactam and ceftazidime. The presence of integrons was also associated with multidrug resistance phenotype. The genetic content of integrons comprised genes determining resistance toward aminoglycosides, sulfonamides and trimethoprim. Moreover, we noticed a
\end{abstract}

Electronic supplementary material The online version of this article (doi:10.1007/s10482-012-9797-9) contains supplementary material, which is available to authorized users.

J. Mokracka $(\bowtie) \cdot$ A. Oszyńska · A. Kaznowski Faculty of Biology, Department of Microbiology, Adam Mickiewicz University in Poznań, ul. Umultowska 89, 61-614 Poznań, Poland

e-mail: amok@amu.edu.pl significant increase in the frequency of $b l a_{\text {CTX-M }}$ $\beta$-lactamases, with appearance of $b l a_{\mathrm{CTX}-\mathrm{M}-15}$ variant and newer plasmid-encoded $\beta$-lactamases like CMY-15 and DHA. The emergence of strains resistant to several classes of antimicrobials and carrying integrons, ESBL and AmpC $\beta$-lactamase-coding genes may predict the spread of isolates with limited treatment options.

Keywords Integrons $\cdot$ ESBL $\cdot$ Antibiotic resistance $\cdot$ ExPEC

\section{Introduction}

Escherichia coli is a facultative Gram-negative species commonly present as a commensal organism in intestinal tract of mammals, but also recognized as one of major pathogens of human and animals. Pathogenic E. coli strains capable of causing disease outside the intestinal tract are classified as extraintestinal pathogenic strains (ExPEC) (Russo and Johnson 2000; Kaper et al. 2004). ExPEC are divided into isolates causing urinary tract and bloodstream infections (uropathogenic strains, UPEC), and neonatal septicemia/meningitis (MNEC, meningitis associated E. coli) strains (Welch 2006). During the past years, the rates of antimicrobial resistance among ExPEC strains have increased substantially, leading to higher morbidity and mortality and substantially increasing treatment costs (Daikos et al. 2007). Cephalosporins, fluoroquinolones, and co-trimoxazole are often used to treat 
ExPEC infections and the surveillance studies in years 2000-2010 indicated that 20-40\% strains become resistant to that group of antimicrobials (Pitout 2012).

Antibiotic resistance may develop through mutations in chromosomal DNA or acquisition of plasmids or transposons carrying resistance determinants. Integrons play an important role in the spread of antimicrobial resistance of clinical Enterobacteriaceae strains, since they capture, integrate and express gene cassettes encoding proteins associated with antimicrobial resistance. The integron covers DNA fragment that consists of an integrase gene of the tyrosine recombinase family, primary recombination site called the attI, and a promoter $\mathrm{P}_{\mathrm{C}}$ that directs transcription of the captured genes (Hall and Collis 1995; Mazel 2006). Integrons are often associated with mobile DNA elements like transposons and plasmids, which enable lateral spread of resistance determinants. Five classes of integrons are recognized on the basis of integrase gene sequence (Cambray et al. 2010). All classes are associated with resistance determinants, three of them are responsible for multidrug resistance (MDR), with class 1 being most ubiquitous among clinical strains (Leverstein-van Hall et al. 2003; Mokracka et al. 2011). Over 130 different gene cassettes have been identified within integrons, providing resistance to most classes of antimicrobials, including $\beta$-lactams, aminoglycosides, amphenicols, macrolides, trimethoprim, quinolones and antiseptics (Partridge et al. 2009; Cambray et al. 2010). Enterobacteriaceae strains harbour numerous integron-embedded antimicrobial resistance determinants, including $\beta$-lactamase-coding genes (Weldhagen 2004; Eckert et al. 2006).

The aim of this research was to analyze the resistance patterns and characterize distribution and genetic content of integrons and $\beta$-lactamase encoding genes within the $E$. coli strains isolated from extraintestinal infections in the course of last decade.

\section{Materials and methods}

\section{Clinical specimens}

Sixty-nine clinically relevant strains of E. coli were isolated from specimens from inpatients of Poznań hospitals. Thirty-eight strains were collected in 1999-2001 (Group 1) and thirty-one from December 2008 to May 2010 (Group 2). The organisms were grown on MacConkey agar no. 3 (Oxoid) and identified with API 20E (bioMérieux) as E. coli. They were isolated from urine (32 strains), throat swab (10), vaginal swab (7), blood (5 strains), broncho-alveolar lavage (4), semen (3), eye ventricle (2), and single strains from cerebrospinal fluid, wound, sputum, tracheostomy tube, ulceration and abscess.

The strains were stored in $-80{ }^{\circ} \mathrm{C}$ in BHI/glycerol (50/50). All phenotypic assays and determination of antimicrobial resistance were done immediately after collection. The interpretation of zone diameters was done according to the CLSI (2009) breakpoints.

\section{Clonal analysis by ERIC-PCR}

The ERIC-PCR method utilizes primers complementary to enterobacterial repetitive intergenic consensus sequences of genomic DNA. The PCR reaction with primers ERIC 1 and ERIC 2 were done according to Versalovic et al. (1991). Computer analysis of electrophoretic patterns was carried out using GelCompar II version 3.5 software (Applied Maths). Similarity between fingerprints was calculated with the Dice coefficient. Cluster analysis was performed using the unweighted pair-group method with average linkages (UPGMA).

\section{Antimicrobial susceptibility}

The susceptibility to 29 antibiotics representing 11 classes was determined according to the standard disc diffusion method recommended by Clinical and Laboratory Standards Institute guidelines (CLSI 2009). The antimicrobials included: amikacin $(30 \mu \mathrm{g})$, tobramycin $(10 \mu \mathrm{g})$, netilmicin $(30 \mu \mathrm{g})$, gentamicin $(10 \mu \mathrm{g})$, kanamycin $(30 \mu \mathrm{g})$, streptomycin $(10 \mu \mathrm{g})$, ampicillin $(10 \mu \mathrm{g})$, ticarcillin $(75 \mu \mathrm{g})$, ciprofloxacin $(5 \mu \mathrm{g})$, norfloxacin $(10 \mu \mathrm{g})$, tetracycline $(30 \mu \mathrm{g})$, amoxicillin/clavulanic acid $(30 \mu \mathrm{g})$, cefotaxime $(30 \mu \mathrm{g})$, ceftazidime $(10 \mu \mathrm{g})$, cefuroxime $(10 \mu \mathrm{g})$, cefoperazone $(10 \mu \mathrm{g})$, cefazolin $(10 \mu \mathrm{g})$, cephalothin $(30 \mu \mathrm{g})$, cefepime $(30 \mu \mathrm{g})$, cefoxitin $(30 \mu \mathrm{g})$, sulfamethoxazole $(25 \mu \mathrm{g})$, co-trimoxazole $(25 \mu \mathrm{g})$, trimethoprim $(5 \mu \mathrm{g})$, piperacillin $(100 \mu \mathrm{g})$, piperacillin/ tazobactam $(110 \mu \mathrm{g})$, chloramphenicol $(30 \mu \mathrm{g})$, aztreonam $(30 \mu \mathrm{g})$, nitrofurantoin $(300 \mu \mathrm{g})$, and imipenem $(10 \mu \mathrm{g})$. All strains exhibiting intermediate resistance zones were considered resistant. Production of extended spectrum $\beta$-lactamases (ESBL) was checked 
by the double-disc synergy test with ceftazidime, cefotaxime and amoxicillin/clavulanic acid.

\section{Identification of bla genes}

Genes coding for extended spectrum $\beta$-lactamases and AmpC lactamases: $b l a_{\mathrm{CTX}-\mathrm{M}}, b l a_{\mathrm{SHV}}, b l a_{\mathrm{TEM}}, b l a_{\mathrm{OXA}}$, $b l a_{\mathrm{GES}}, b l a_{\mathrm{VEB}}, b l a_{\mathrm{PER}}, b l a_{\mathrm{DHA}}$ and $b l a_{\mathrm{CMY}}$ were identified by PCR method. The sequences of primers targeting $\beta$-lactamase genes were published elsewhere (Li CR et al. 2003; Li Y et al. 2008; Sáenz et al. 2004; Mendonça et al. 2008; De Champs et al. 2002; Aragón et al. 2008; Gniadkowski et al. 1998a, b). PCR amplifications were performed in a $25-\mu \mathrm{l}$ volume with $2.5 \mu \mathrm{l}$ of $10 \times$ PCR buffer with $\mathrm{NH}_{4}\left(\mathrm{SO}_{4}\right)_{2}, 0.6 \mu \mathrm{M}$ of each primer, $100 \mu \mathrm{M}$ of dNTP mix, $2.5 \mathrm{mM}$ of $\mathrm{MgCl}_{2}$, $1 \mathrm{U}$ of Hi-Fi Taq polymerase (Novazym), and $200 \mathrm{ng}$ of genomic DNA. Amplification involved an initial denaturation $\left(94{ }^{\circ} \mathrm{C}, 5 \mathrm{~min}\right)$ followed by 30 cycles of denaturation $\left(94{ }^{\circ} \mathrm{C}, 30 \mathrm{~s}\right)$, annealing $\left(55^{\circ} \mathrm{C}, 30 \mathrm{~s}\right.$ for $b l a_{\mathrm{CTX}-\mathrm{M}}$ and $b l a_{\mathrm{SHV}}, 50{ }^{\circ} \mathrm{C} 1 \mathrm{~min}$ for $b l a_{\mathrm{TEM}}$, bla $a_{\mathrm{OXA}}$, bla $a_{\mathrm{GES}}$, bla $\mathrm{VEB}, b l a_{\mathrm{CMY}}, 48{ }^{\circ} \mathrm{C} 1 \mathrm{~min}$ for $b l a_{\mathrm{PER}}$, and $60{ }^{\circ} \mathrm{C} 30 \mathrm{~s}$ for $\left.b l a_{\mathrm{DHA}}\right)$, and extension $\left(72{ }^{\circ} \mathrm{C}, 1 \mathrm{~min}\right)$, with a final extension step $\left(72{ }^{\circ} \mathrm{C}\right.$, $8 \mathrm{~min})$. Variants of $b l a_{\mathrm{TEM}}, b l a_{\mathrm{CTX}-\mathrm{M}}, b l a_{\mathrm{OXA}}, b l a_{\mathrm{SHV}}$, $b l a_{\mathrm{CMY}}$, and $b l a_{\mathrm{DHA}}$ were identified upon comparing the sequences with available GenBank sequence data by using ClustalW and the neighbor-joining method.

The genetic environment of $b l a_{\text {СТХ-M }}$ was determined with primers sets targeting ISCR1 (formerly orf513), and ISEcpl (Eckert et al. 2006; Quiroga et al. 2007).

\section{Analysis of integrons}

Multiplex PCR was done for identification of integron integrase genes. PCR amplification was performed in a $25-\mu \mathrm{l}$ volume with $2.5 \mu \mathrm{l}$ of $10 \times$ PCR buffer with $\mathrm{NH}_{4}\left(\mathrm{SO}_{4}\right)_{2}, 0.25 \mu \mathrm{M}$ of each primer, $100 \mu \mathrm{M}$ of dNTP mix, $2.5 \mathrm{mM}$ of $\mathrm{MgCl}_{2}, 1 \mathrm{U}$ of Hi-Fi Taq polymerase, and $200 \mathrm{ng}$ of genomic DNA. Amplification involved an initial denaturation $\left(94{ }^{\circ} \mathrm{C}, 5 \mathrm{~min}\right)$ followed by 30 cycles of denaturation $\left(94^{\circ} \mathrm{C}, 1 \mathrm{~min}\right)$, annealing $\left(59^{\circ} \mathrm{C}, 1 \mathrm{~min}\right)$ and extension $\left(72{ }^{\circ} \mathrm{C}\right.$, $1 \mathrm{~min})$, with a final extension step $\left(72{ }^{\circ} \mathrm{C}, 8 \mathrm{~min}\right)$. The sequences of primers targeting intIl, intI 2 and intI3 genes were recommended by Dillon et al. (2005).
Variable regions of class 1 and class 2 integrons were analyzed by conserved segment PCR (CS-PCR) and Hep-PCR, respectively. Sequences of primers complementary to the $5^{\prime}$ and $3^{\prime}$ conserved regions of 1 class integrons ( $5^{\prime}-\mathrm{CS}$ and $3^{\prime}$-CS) and class 2 integron (Hep74 and Hep51) were published elsewhere (Lévesque et al. 1995; White et al. 2001). PCR amplifications were conducted as follow: initial denaturation $94{ }^{\circ} \mathrm{C}, 5 \mathrm{~min}$, and 30 cycles of $94{ }^{\circ} \mathrm{C} 1 \mathrm{~min}$, $55^{\circ} \mathrm{C} 1 \mathrm{~min}, 72{ }^{\circ} \mathrm{C} 5 \mathrm{~min}$, and final elongation $72{ }^{\circ} \mathrm{C} 8 \mathrm{~min}$.

To check the gene cassette content, CS-PCR and Hep-PCR products were purified and sequenced. In case of two amplicons and amplicons longer than $1.7 \mathrm{kbp}$, the products were cloned by using $\mathrm{pGEM}^{\circledR}-\mathrm{T}$ Easy Vector (Promega). Sequence data were analyzed with DNA Baser (HeracleSoftware) and aligned with available GenBank data using BLASTn. A gene cassette was identified if the percentage of similarity with GenBank data was higher than $95 \%$.

All PCR reactions were performed in a $\mathrm{C} 1000$ Thermal Cycler (BioRad). The PCR products were separated in $1.5 \%$ agarose gel (Novazym). Molecular weight of PCR products was determined by Bio-Capt v. 99.04 software (Vilber Lourmat). All experiments were done in triplicate.

\section{Statistical analysis}

Association between the frequency of antibiotic resistance and integron presence was calculated using Pearson's $\chi^{2}$ test and Fisher's exact test. Association between integron presence and resistance ranges was determined with the Mann-Whitney $U$ test (Statistica 10, StatSoft). $P<0.05$ was considered to indicate statistical significance.

\section{Results}

\section{Clonal analysis by ERIC-PCR}

The fingerprints of E. coli isolates consisted of 1-17 bands ranging in size from 110 to 5,000 bp (Supplementary Fig. S1). Three pair of isolates (EC1 and EC3, EC45 and EC53, EC49 and EC62) had ERIC-PCR profiles with $100 \%$ similarity, yet analysis of integrons and resistance patterns indicated differences 
between them. Strains EC1 and EC3 had different resistance patterns, EC45 and EC53 differed in bla gene content, whereas EC49 and EC62 had also different integron gene cassette arrays (Table 1).

Antimicrobial susceptibility

Strains isolated in 1999-2001 were resistant towards 1-22 antimicrobials (median 7); the highest frequency of resistance was noted to streptomycin $(86.8 \%)$, sulfamethoxazole $(86.6 \%)$ cephalotin $(57.9 \%)$ and tetracycline $(55.3 \%)$, the lowest to imipenem (0\%), norfloxacin $(5.3 \%)$, pipercillin/tazobactam and chloramphenicol $(7.9 \%)$. The percentage of ESBL-producing isolates was $21.1 \%$. The frequency of resistance of strains isolated in the beginning and in the end of the decade is shown in Fig. 1.

Strains isolated in 2008-2010 had significantly broader resistance ranges defined as the number of antimicrobials to which isolates were resistant and the number of antimicrobial classes to which isolates were resistant (Mann-Whitney $U$ test, $P<0.001$ ) (Fig. 2). They were resistant towards 4-28 antimicrobials (median 22). The highest resistance percentage was noted for sulfamethoxazole and ampicillin (100\%), streptomycin, cephalothin, cefoperazone, cefuroxime, cefazolin, cephalothin and cefepim $(96.8 \%)$. The lowest levels of resistance were recorded for imipenem $(0 \%)$, pipercillin/tazobactam $(22.6 \%)$ and nitrofurantoin $(35.5 \%)$. The percentage of ESBL producers reached $93.5 \%$.

The frequency of resistance to each of the antimicrobials beside imipenem, streptomycin, piperacillin/ tazobactam, and sulfamethoxazole was significantly higher in the second group of isolates $(P<0.05)$.

\section{Analysis of integrons}

We detected integrase genes in $53.6 \%$ isolates (37 strains); 35 had intIl gene, one intI2 gene and one strain had both intII and intI2 genes (Table 1). We found class 1 integrons in the genomes of $31.6 \%$ strains isolated in 1999-2001, compared to $80.7 \%$ strains isolated in 2008-2010. The frequency of int positive strains was significantly lower in the first group of isolates $(P<0.001)$. We amplified variable regions of class 1 integrons and detected amplicons of the following sizes: $0.55 \mathrm{kbp}$ (1 strain), $0.7 \mathrm{kbp}(4)$, $1.6 \mathrm{kbp}(7), 1.7 \mathrm{kbp}(6), 1.9 \mathrm{kbp}$ (11), and $3.0 \mathrm{kbp}(1)$. Two strains had two integrons: 1.0 and $1.7 \mathrm{kbp}$, and 1.6 and $3.0 \mathrm{kbp}$ (Table 1). We did not manage to receive a CS-PCR product for four intIl-positive strains. The two class 2 integrons had variable regions of $2.2 \mathrm{kbp}$.

The sequence analysis of integrons' variable regions showed the presence of 1-5 genes. The most often identified gene cassettes were: aminoglycoside adenylyltransferase $\operatorname{aadA}(\operatorname{aad} A 1,2,5)$ conferring resistance to aminoglycosides: streptomycin and

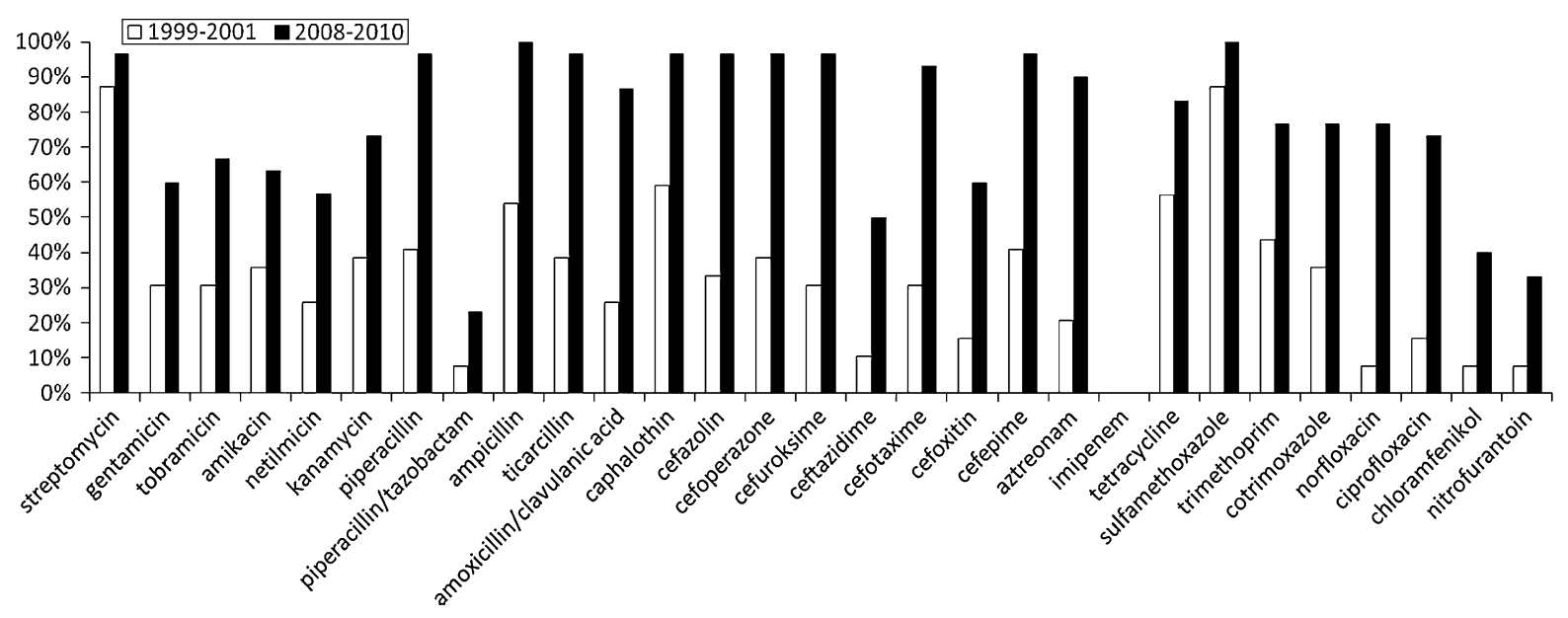

Fig. 1 The frequency of antibiotic resistance of E. coli strains isolated in 1999-2001 and 2008-2010 

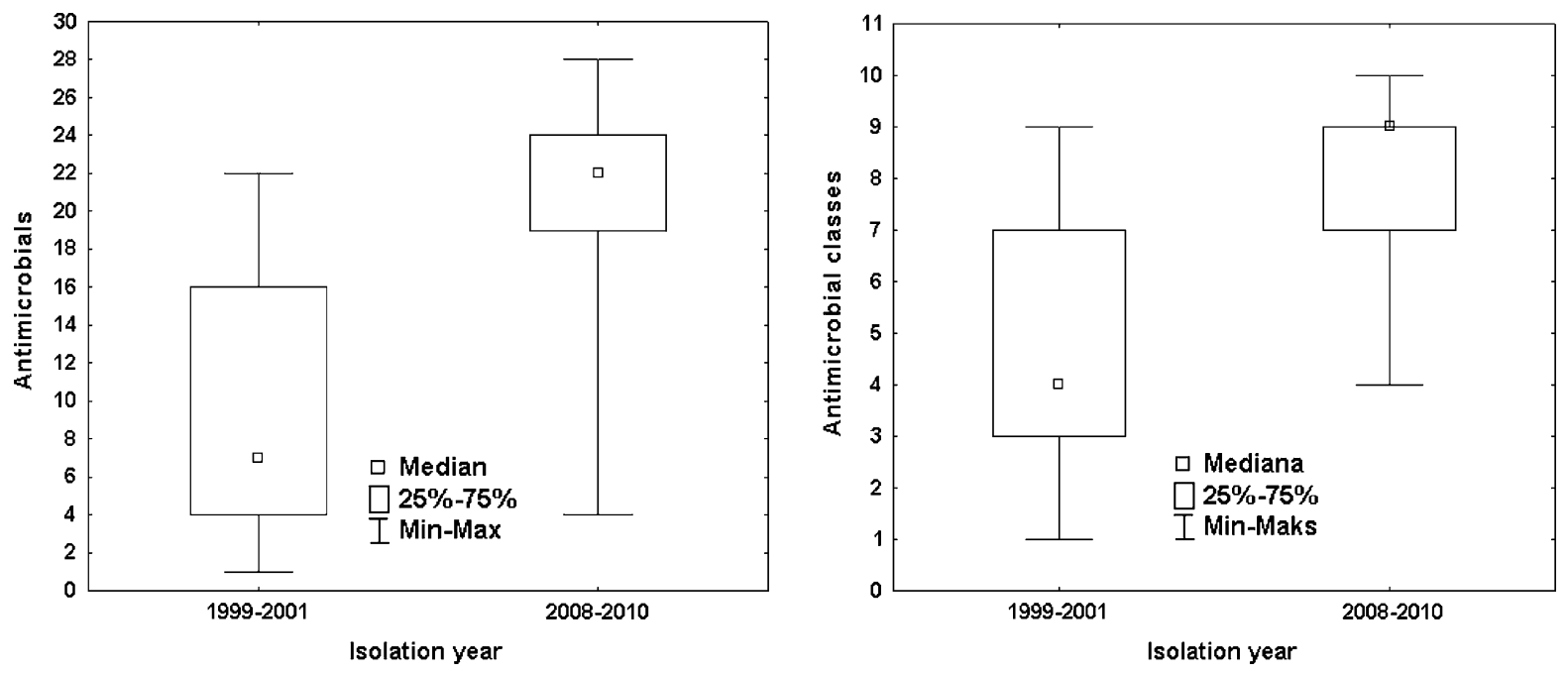

Fig. 2 Box plots representing antimicrobial resistance ranges of E. coli strains isolated in 1999-2001 and 2008-2010

spectinomycin, aminoglycoside acetyltransferases, aacA4 and aacC1, responsible for resistance to tobramycin, amikacin and gentamicin, respectively, dihydrofolate reductase $d f r A(d f r A 1,12,17, d f r 2 d)$ conferring resistance to trimethoprim, and streptothricin acetyltransferase sat 2 conferring resistance to streptothricin. We detected the following gene cassette arrays within class 1 integrons: $d f r 2 d(0.55 \mathrm{kbp})$, aadAl $(1.0 \mathrm{kbp})$, both present as a single cassette within integron, dfrAl-aadAl (1.6 kbp), dfrAl7aadA5 (1.7 kbp), dfrA12-orfF-aadA2 (1.9 kbp), and aacA4-aacC1-orfX-orfY-aadA1 (3.0 kbp). Class 2 integron present in the genomes of two strains had dfrAl-sat2-aadAl arrays. In four strains we detected amplicons of $0.7 \mathrm{kbp}$ which were in $99 \%$ identical to a gene coding for hypothetical protein in multiresistant uropathogenic E. coli isolated in India (Acc. No CP002797.2) (Table 1).

\section{Presence of bla genes}

The strains were examined for presence of genes coding for $\beta$-lactamases. Altogether, we identified the following bla genes: bla $a_{\mathrm{CTX}-\mathrm{M}-1}$-like gene (28 strains), $b l a_{\mathrm{TEM}}(20), b l a_{\mathrm{OXA}}$ (7) and $b l a_{\mathrm{SHV}}$ (5). Sequencing of the amplicons and comparing against GenBank databases identified bla genes as $b l a_{\mathrm{CTX-M-1}}, b l a_{\mathrm{CTX-М-3}}$, bla $a_{\mathrm{CTX}-\mathrm{M}-15}, b l a_{\mathrm{CTX}-\mathrm{M}-55}, b l a_{\mathrm{TEM}-1}, b l a_{\mathrm{TEM}-2}, b l a_{\mathrm{OXA}-1}$, $b l a_{\mathrm{SHV}-1}$, and $b l a_{\mathrm{SHV}-12}$. Strains harbouring $b l a_{\mathrm{CTX}-\mathrm{M}}$ had broader resistance ranges than those without $b l a_{\text {СтХ-м }}(P<0.001)$. The distribution of bla genes is shown in Table 1.

Eight strains $(21.0 \%)$ isolated in 1999-2001 harboured $b l a_{\mathrm{CTX}-\mathrm{M}-3}$. Besides, six strains had $b l a_{\mathrm{TEM}-1}$ gene and one isolate $b l a_{\mathrm{SHV}-1}$. In the second group, bla genes were found in 29 isolates $(96.7 \%)$ and identified as $b l a_{\mathrm{CTX}-\mathrm{M}-3}$ (3 isolates), $b l a_{\mathrm{CTX}-\mathrm{M}-15}$ (2), bla $a_{\mathrm{CTX}-\mathrm{M}-1}$ (1), bla $a_{\mathrm{CTX}-\mathrm{M}-55}$ (1), bla ${ }_{\mathrm{TEM}-1}$ (5), $b l a_{\mathrm{OXA}-1}(1)$ and $b_{\text {SHV-12 }}$ (1) as single bla genes. Eleven ESBL-producing isolates had two bla genes whereas three isolates had three bla genes (Table 1). In 24 strains $(86 \%)$, the genes coding for CTX-M $\beta$-lactamase were associated with ISEcpl which was found upstream a $b l a_{\text {СтХ-M }}$ gene. The presence of ESBL-producing strains was associated with the time of isolation; there were significantly less ESBL producers among isolates from 1999 to 2001 $(P<0.001)$ and they had significantly lower number of bla $_{\mathrm{CTX-M}}(P<0.001)$, bla $a_{\mathrm{TEM}}(P=0.015)$ and $b_{\text {bla }}(P=0.002)$ genes. The presence of ESBL phenotype, and ESBL encoding genes $\left(b l a_{\mathrm{CTX}-\mathrm{M}}\right.$ and $\left.b l a_{\mathrm{SHV}}\right)$ was associated with the presence of integrons $(P<0.001)$.

We found AmpC cephalosporinase genes in five strains: four of them had $b l a_{\mathrm{CMY}}$ and one $b l a_{\mathrm{DHA}}$. Sequencing of $b l a_{\mathrm{CMY}}$ and comparing the sequences versus available GenBank data identified them as $b l a_{\mathrm{CMY}-15}$. The $b l a_{\mathrm{CMY}}$ and $b l a_{\mathrm{DHA}}$ genes were present in isolates that had $b l a_{\text {CTX-M }}$ and integrons. 
Table 1 Integrons, $\beta$-lactamase-coding genes and resistance patterns of E. coli isolates

\begin{tabular}{|c|c|c|c|c|c|c|}
\hline Strain & Origin & Integrase & $\begin{array}{l}\text { Integron } \\
\text { variable } \\
\text { region size } \\
(\mathrm{kbp})\end{array}$ & $\begin{array}{l}\text { Integron's } \\
\text { gene } \\
\text { cassettes }\end{array}$ & bla genes & Resistance pattern \\
\hline \multicolumn{7}{|c|}{ 1999-2001 (38 isolates) } \\
\hline $\mathrm{EC} 1$ & Urine & - & - & - & - & KAN, STR, AMP, FOX, CEF, SUL, ATM \\
\hline EC2 & Blood & - & - & - & - & STR, CEF, SUL \\
\hline EC3 & Urine & - & - & - & - & $\begin{array}{l}\text { KAN, STR, AMP, FEP, FOX, CEF, SUL, SXT, } \\
\text { CHL }\end{array}$ \\
\hline EC4 & Blood & intI1 & 1.6 & $\begin{array}{l}\text { dfrAl- } \\
\text { aadAl }\end{array}$ & $b l a_{\mathrm{TEM}-1}$ & $\begin{array}{l}\text { AMK, GEN, STR, AMP, PIP, TIC, AMC, FEP, } \\
\text { CFP, CXM, CEF, SUL, TMP, SXT, TET, CHL }\end{array}$ \\
\hline EC5 & $\begin{array}{l}\text { Cerebrospinal } \\
\text { fluid }\end{array}$ & - & - & - & - & GEN, STR, AMP, TZP, FEP, CEF, SUL, TET \\
\hline EC6 & Blood & intI1 & 1.7 & $\begin{array}{r}d f r A 17- \\
\text { aadA5 }\end{array}$ & $b l a_{\mathrm{TEM}-1}$ & $\begin{array}{l}\text { AMK, GEN, NET, TOB, STR, AMP, PIP, TIC, } \\
\text { AMC, FEP, CFP, FOX, CXM, CEF, SUL, } \\
\text { TMP, SXT, TET, CHL, NIT }\end{array}$ \\
\hline EC7 & $\begin{array}{l}\text { Eye ventricle } \\
\text { swab }\end{array}$ & - & - & - & - & $\begin{array}{l}\text { NET, STR, AMP, TZP, TIC, FEP, CTX, CAZ, } \\
\text { CEF, CIP, TMP, TET, NIT }\end{array}$ \\
\hline EC8 & Urine & - & - & - & $b l a_{\mathrm{TEM}-1}$ & $\begin{array}{l}\text { STR, AMP, PIP, TIC, AMC, CFZ, CFP, CTX, } \\
\text { FOX, CEF, SUL }\end{array}$ \\
\hline EC9 & Urine & intI1 & 1.9 & $\begin{array}{l}\text { dfrA12- } \\
\text { orfF- } \\
\quad \operatorname{aadA2}\end{array}$ & $b l a_{\mathrm{CTX}-\mathrm{M}-3}$ & $\begin{array}{l}\text { AMK, GEN, KAN, NET, STR, TOB, AMP, PIP, } \\
\text { TIC, AMC, CFZ, FEP, CFP, CTX, CXM, CEF, } \\
\text { SUL, TMP, SXT, TET, ATM }\end{array}$ \\
\hline EC10 & Vaginal swab & - & - & - & - & AMK, GEN, STR, FOX, SUL \\
\hline EC11 & Urine & - & - & - & - & STR, AMP, FEP, CFP, CEF, SUL, TMP \\
\hline EC12 & Blood & - & - & - & $b l a_{\mathrm{TEM}-1}$ & $\begin{array}{l}\text { STR, AMP, PIP, TIC, AMC, CFZ, CFP, CTX, } \\
\text { CEF, TET }\end{array}$ \\
\hline EC13 & Urine & - & - & - & - & STR, TET \\
\hline EC14 & Urine & - & - & - & - & STR, SUL \\
\hline EC15 & Urine & - & - & - & - & KAN, STR, SUL \\
\hline EC16 & Urine & - & - & - & - & STR, PIP, SUL, TET \\
\hline EC17 & Urine & - & - & - & - & AMK, TOB, CAZ, CEF, SUL \\
\hline EC18 & Semen & - & - & - & $b l a_{\mathrm{TEM}-1}$ & STR, AMP, TIC, SUL, TET \\
\hline EC19 & Urine & - & - & - & - & CEF, CIP, SUL \\
\hline EC20 & Semen & - & - & - & - & STR, CIP, NOR, SUL \\
\hline EC21 & Urine & - & - & - & - & STR, TOB, AMP, CEF, SUL, TMP \\
\hline EC22 & Urine & - & - & - & - & STR, CFZ, CIP, SUL \\
\hline EC23 & Semen & - & - & - & - & TZP, FEP \\
\hline $\mathrm{EC} 24$ & Urine & - & - & - & $b l a_{\mathrm{TEM}-1}$ & AMK, STR, TOB, CXM, SUL, TET \\
\hline $\mathrm{EC} 25$ & Urine & - & - & - & - & STR, SUL, TET \\
\hline EC26 & $\begin{array}{l}\text { Eye ventricle } \\
\text { swab }\end{array}$ & - & - & - & - & STR, SUL, TET \\
\hline $\mathrm{EC} 27$ & Brain abscess & - & - & - & $b l a_{\mathrm{SHV}-1}$ & AMP, PIP, TIC, CAZ, SUL \\
\hline EC28 & Throat swab & intII & 1.6 & $\begin{array}{l}\text { dfrAl- } \\
\text { aadAl }\end{array}$ & - & KAN, STR, PIP, CFZ, SUL, TMP, SXT \\
\hline EC29 & Throat swab & - & - & - & - & KAN, STR, PIP, AMC, CEF, SUL, TMP, TET \\
\hline EC30 & Urine & intI1 & 1.6 & $\begin{array}{l}\text { dfrAl- } \\
\text { aadAl }\end{array}$ & - & AMK, KAN, STR, CFP, SUL, TMP, SXT \\
\hline
\end{tabular}


Table 1 continued

\begin{tabular}{|c|c|c|c|c|c|c|}
\hline Strain & Origin & Integrase & $\begin{array}{l}\text { Integron } \\
\text { variable } \\
\text { region size } \\
(\mathrm{kbp})\end{array}$ & $\begin{array}{l}\text { Integron's } \\
\text { gene } \\
\text { cassettes }\end{array}$ & bla genes & Resistance pattern \\
\hline EC31 & Urine & intII & 1.9 & $\begin{array}{l}\text { dfrA12- } \\
\text { orfF- } \\
\text { aadA2 }\end{array}$ & $b l a_{\mathrm{CTX}-\mathrm{M}-3}$ & $\begin{array}{l}\text { AMK, GEN, KAN, NET, STR, TOB, AMP, PIP, } \\
\text { TIC, AMC, CFZ, FEP, CFP, CTX, CXM, CEF, } \\
\text { SUL, TMP, SXT, TET, ATM }\end{array}$ \\
\hline EC32 & Throat swab & intII & 1.9 & $\begin{array}{l}\text { dfrA } 12- \\
\text { orfF- } \\
\quad \operatorname{aadA2}\end{array}$ & $b l a_{\mathrm{CTX}-\mathrm{M}-3}$ & $\begin{array}{l}\text { AMK, GEN, KAN, NET, STR, TOB, AMP, PIP, } \\
\text { TIC, CFZ, FEP, CFP, CTX, CXM, CEF, SUL, } \\
\text { TMP, SXT, TET }\end{array}$ \\
\hline EC33 & Urine & intI1 & 1.9 & $\begin{array}{l}\text { dfrA } 12- \\
\text { orfF- } \\
\text { aadA2 }\end{array}$ & $b l a_{\mathrm{CTX}-\mathrm{M}-3}$ & $\begin{array}{l}\text { AMK, GEN, KAN, NET, STR, TOB, AMP, PIP, } \\
\text { TIC, CFZ, FEP, CFP, CTX, CXM, CEF, CIP, } \\
\text { SUL, TMP, SXT, TET, ATM }\end{array}$ \\
\hline EC34 & Throat swab & intII & 1.9 & $\begin{array}{l}\text { dfrA } 12- \\
\text { orfF- } \\
\quad \operatorname{aadA2}\end{array}$ & $b l a_{\mathrm{CTX}-\mathrm{M}-3}$ & $\begin{array}{l}\text { AMK, GEN, KAN, NET, STR, TOB, AMP, PIP, } \\
\text { TIC, CFZ, FEP, CFP, CTX, CXM, CEF, SUL, } \\
\text { TMP, SXT, TET, ATM }\end{array}$ \\
\hline EC35 & Throat swab & intII & 1.9 & $\begin{array}{l}\text { dfrA } 12- \\
\text { orfF- } \\
\quad \operatorname{aadA2}\end{array}$ & $b l a_{\mathrm{CTX}-\mathrm{M}-3}$ & $\begin{array}{l}\text { AMK, GEN, KAN, NET, STR, TOB, AMP, PIP, } \\
\text { TIC, AMC, CFZ, FEP, CFP, CTX, CXM, CEF, } \\
\text { NOR, SUL, TMP, SXT, TET, ATM }\end{array}$ \\
\hline EC36 & Throat swab & intIl & 1.9 & $\begin{array}{l}\text { dfrA12- } \\
\text { orfF- } \\
\text { aadA2 }\end{array}$ & $b l a_{\mathrm{CTX}-\mathrm{M}-3}$ & $\begin{array}{l}\text { AMK, GEN, KAN, NET, STR, TOB, AMP, PIP, } \\
\text { TIC, AMC, CFZ, FEP, CFP, CTX, CXM, CEF, } \\
\text { SUL, TMP, SXT, TET }\end{array}$ \\
\hline EC37 & Throat swab & intII & 1.9 & $\begin{array}{l}\text { dfrA12- } \\
\text { orfF- } \\
\text { aadA2 }\end{array}$ & $b l a_{\mathrm{CTX}-\mathrm{M}-3}$ & $\begin{array}{l}\text { AMK, GEN, KAN, NET, STR, TOB, AMP, PIP, } \\
\text { TIC, CFZ, FEP, CFP, CTX, CXM, CEF, SUL, } \\
\text { TMP, SXT, TET, ATM }\end{array}$ \\
\hline EC38 & Throat swab & - & - & - & - & STR, TET \\
\hline \multicolumn{7}{|c|}{ 2008-2010 (31 isolates) } \\
\hline EC39 & Vaginal swab & intI2 & 2.2 & $\begin{array}{l}\text { dfrAl- } \\
\quad \text { sat1- } \\
\quad \text { aadAl }\end{array}$ & $\begin{array}{r}b^{b} a_{\mathrm{CTX}-\mathrm{M}-1} \\
b l a_{\mathrm{CMY}-15}\end{array}$ & $\begin{array}{l}\text { KAN, STR, AMP, AMC, CFZ, FEP, CFP, CTX, } \\
\text { FOX, CAZ, CXM, CEF, CIP, NOR, SUL, } \\
\text { TMP, SXT, TET, ATM, NIT }\end{array}$ \\
\hline EC40 & $\begin{array}{l}\text { Tracheostomy } \\
\text { tube }\end{array}$ & - & - & - & - & STR, AMP, CIP, SUL \\
\hline EC41 & Wound & intI1 & 1.9 & $\begin{array}{l}\text { dfrA } 12- \\
\text { orfF- } \\
\text { aadA2 }\end{array}$ & $\begin{array}{c}b^{b l a_{\mathrm{CTX}-\mathrm{M}-3}}, \\
\text { bla }_{\mathrm{TEM}-1}\end{array}$ & $\begin{array}{l}\text { AMK, GEN, KAN, NET, STR, TOB, AMP, PIP, } \\
\text { TZP, TIC, AMC, CFZ, FEP, CFP, CTX, FOX, } \\
\text { CXM, CEF, SUL, TMP, SXT, TET, ATM }\end{array}$ \\
\hline EC42 & Urine & intII & 0.55 & $d f r 2 d$ & $\begin{array}{l}\text { bla }_{\mathrm{CTX}-\mathrm{M}-3} \\
\text { bla }_{\mathrm{TEM}-1} \\
\text { bla }_{\mathrm{DHA}}\end{array}$ & $\begin{array}{l}\text { AMK, GEN, KAN, NET, STR, TOB, AMP, PIP, } \\
\text { TZP, TIC, AMC, CFZ, FEP, CFP, CTX, FOX, } \\
\text { CXM, CEF, CIP, NOR, SUL, TMP, SXT, TET, } \\
\text { ATM, CHL }\end{array}$ \\
\hline EC43 & Throat swab & intII & 1.9 & $\begin{array}{l}\text { dfrA12- } \\
\text { orfF- } \\
\quad \operatorname{aadA2}\end{array}$ & $b l a_{\mathrm{CTX}-\mathrm{M}-3}$ & $\begin{array}{l}\text { AMK, GEN, KAN, NET, STR, TOB, AMP, PIP, } \\
\text { TIC, AMC, CFZ, FEP, CFP, CTX, CXM, CEF, } \\
\text { SUL, TMP, SXT, TET, ATM }\end{array}$ \\
\hline EC44 & $\begin{array}{l}\text { Broncho- } \\
\text { alveolar } \\
\text { lavage }\end{array}$ & intII & n.d. & n.d. & $b l a_{\mathrm{TEM}-1}$ & $\begin{array}{l}\text { AMK, GEN, KAN, NET, STR, TOB, AMP, PIP, } \\
\text { TZP, TIC, AMC, CFZ, FEP, CFP, CTX, CAZ, } \\
\text { CXM, CEF, SUL, SXT, TET, ATM }\end{array}$ \\
\hline EC45 & $\begin{array}{l}\text { Broncho- } \\
\text { alveolar } \\
\text { lavage }\end{array}$ & intII & 1.6 & $\begin{array}{l}d f r A 1- \\
\quad \operatorname{aadA1} 1\end{array}$ & $\begin{array}{r}b^{b l a_{\mathrm{SHV}-12}}, \\
\text { bla }_{\mathrm{TEM}-1}\end{array}$ & $\begin{array}{l}\text { AMK, GEN, KAN, NET, STR, TOB, AMP, PIP, } \\
\text { TIC, AMC, CFZ, FEP, CFP, CTX, CAZ, } \\
\text { CXM, CEF, CIP, NOR, SUL, TMP, SXT, TET, } \\
\text { ATM, NIT }\end{array}$ \\
\hline EC46 & Throat swab & intII & 1.9 & $\begin{array}{l}d f r A 12- \\
\text { orfF- } \\
\quad \operatorname{aadA2}\end{array}$ & bla $_{\mathrm{SHV}-12}$ & $\begin{array}{l}\text { AMK, GEN, KAN, NET, STR, TOB, AMP, PIP, } \\
\text { TIC, AMC, CFZ, FEP, CFP, CTX, CAZ, } \\
\text { CXM, CEF, SUL, TMP, SXT, TET, ATM, } \\
\text { CHL, NIT }\end{array}$ \\
\hline
\end{tabular}


Table 1 continued

\begin{tabular}{|c|c|c|c|c|c|c|}
\hline Strain & Origin & Integrase & $\begin{array}{l}\text { Integron } \\
\text { variable } \\
\text { region size } \\
(\mathrm{kbp})\end{array}$ & $\begin{array}{l}\text { Integron's } \\
\text { gene } \\
\text { cassettes }\end{array}$ & bla genes & Resistance pattern \\
\hline EC47 & Urine & intI1 & n.d. & n.d. & $b l a_{\mathrm{TEM}-1}$ & $\begin{array}{l}\text { STR, AMP, PIP, TIC, AMC, CFZ, FEP, } \\
\text { CFP, CTX, CXM, CEF, CIP, NOR, SUL, } \\
\text { TMP, SXT, TET, ATM, CHL }\end{array}$ \\
\hline EC48 & $\begin{array}{l}\text { Vaginal } \\
\text { swab }\end{array}$ & intI1 & 1.7 & $d f r A 17-a a d A 5$ & $\begin{array}{l}\text { bla }_{\mathrm{CTX}-\mathrm{M}-15} \\
\text { bla } \\
\text { blaM-1 } \\
\text { bla } \\
\mathrm{OXA}-1\end{array}$ & $\begin{array}{l}\text { STR, AMP, PIP, TIC, AMC, CFZ, FEP, } \\
\text { CFP, CTX, CAZ, CXM, CEF, CIP, NOR, } \\
\text { SUL, TMP, SXT, TET, CHL, NIT }\end{array}$ \\
\hline EC49 & $\begin{array}{l}\text { Vaginal } \\
\text { swab }\end{array}$ & intI1 & 1.6 & $d f r A 1-a a d A 1$ & $\begin{array}{l}\text { bla }_{\mathrm{CTX}-\mathrm{M}-15}, \\
\text { bla }_{\mathrm{TEM}}\end{array}$ & $\begin{array}{l}\text { STR, AMP, PIP, TZP, TIC, AMC, CFZ, } \\
\text { FEP, CFP, CTX, CXM, CEF, CIP, NOR, } \\
\text { SUL, TMP, SXT, TET, ATM }\end{array}$ \\
\hline EC50 & Ulceration & intI1 & 1.7 & dfrA17-aadA5 & $b l a_{\mathrm{CTX}-\mathrm{M}-15}$ & $\begin{array}{l}\text { GEN, KAN, STR, TOB, AMP, PIP, TIC, } \\
\text { AMC, CFZ, FEP, CFP, CTX, FOX, } \\
\text { CAZ, CXM, CEF, CIP, NOR, SUL, } \\
\text { TMP, SXT, TET, ATM, CHL }\end{array}$ \\
\hline EC51 & Urine & intI1 & $1.0+1.7$ & $\begin{array}{l}a a d A 1+d f r A 17- \\
\quad \text { aadA5 }\end{array}$ & $\begin{array}{c}b^{b l a_{\mathrm{CTX}-\mathrm{M}-15}}, \\
\text { bla }_{\mathrm{OXA}-1}\end{array}$ & $\begin{array}{l}\text { AMK, GEN, KAN, NET, STR, TOB, } \\
\text { AMP, PIP, TZP, TIC, AMC, CFZ, FEP, } \\
\text { CFP, CTX, FOX, CAZ, CXM, CEF, CIP, } \\
\text { NOR, SUL, TMP, SXT, TET, ATM, } \\
\text { CHL, NIT }\end{array}$ \\
\hline EC52 & $\begin{array}{l}\text { Vaginal } \\
\text { swab }\end{array}$ & - & - & - & $b l a_{\mathrm{TEM}-1}$ & $\begin{array}{l}\text { AMK, KAN, STR, AMP, PIP, TIC, AMC, } \\
\text { CFZ, FEP, CFP, CTX, FOX, CXM, CEF, } \\
\text { CIP, NOR, SUL, ATM }\end{array}$ \\
\hline EC53 & $\begin{array}{l}\text { Bbroncho- } \\
\text { alveolar } \\
\text { lavage }\end{array}$ & intI1 & 1.6 & $d f r A 1-a a d A 1$ & $\begin{array}{l}\text { bla }_{\mathrm{CTX}-\mathrm{M}-15} \\
\text { bla }_{\mathrm{SHV}-12} \\
\text { bla }_{\mathrm{TEM}-1}\end{array}$ & $\begin{array}{l}\text { AMK, GEN, KAN, NET, STR, TOB, } \\
\text { AMP, PIP, TIC, AMC, CFZ, FEP, CFP, } \\
\text { FOX, CAZ, CXM, CEF, CIP, NOR, } \\
\text { SUL, TMP, SXT, TET, ATM, NIT }\end{array}$ \\
\hline EC54 & Urine & intI1 & 1.7 & dfrA17-aadA5 & $b l a_{\mathrm{TEM}-1}$ & $\begin{array}{l}\text { GEN, KAN, STR, TOB, AMP, PIP, TIC, } \\
\text { AMC, CFZ, FEP, CFP, CTX, FOX, } \\
\text { CXM, CEF, CIP, NOR, SUL, TMP, } \\
\text { SXT, TET, ATM, CHL }\end{array}$ \\
\hline EC55 & Urine & - & - & - & - & $\begin{array}{l}\text { STR, AMP, PIP, TIC, AMC, CFZ, FEP, } \\
\text { CFP, CTX, FOX, CXM, CEF, NOR, } \\
\text { SUL, TMP, SXT, TET, ATM, CHL }\end{array}$ \\
\hline EC56 & $\begin{array}{l}\text { Broncho- } \\
\text { alveolar } \\
\text { lavage }\end{array}$ & intI1 & $3.0+1.6$ & $\begin{array}{l}\text { aacA4-aacC1- } \\
\text { orfX-orfY- } \\
\operatorname{aadA1}+d f r A 1- \\
\text { aadA1 }\end{array}$ & $\begin{array}{l}\text { bla }_{\mathrm{CTX-M-3}} \\
\text { bla }_{\mathrm{SHV}-12} \\
\text { bla }_{\mathrm{TEM}-1}\end{array}$ & $\begin{array}{l}\text { AMK, GEN, KAN, NET, STR, TOB, } \\
\text { AMP, PIP, TIC, AMC, CFZ, FEP, CFP, } \\
\text { CTX, FOX, CAZ, CXM, CEF, CIP, } \\
\text { NOR, SUL, TMP, SXT, TET, ATM, } \\
\text { CHL, NIT }\end{array}$ \\
\hline EC57 & Urine & - & - & - & - & $\begin{array}{l}\text { GEN, KAN, STR, AMP, PIP, TIC, AMC, } \\
\text { CFZ, FEP, CFP, CTX, FOX, CXM, CEF, } \\
\text { SUL, ATM }\end{array}$ \\
\hline EC58 & $\begin{array}{c}\text { Vaginal } \\
\text { swab }\end{array}$ & - & - & - & $b l a_{\mathrm{TEM}-1}$ & $\begin{array}{l}\text { AMP, PIP, TZP, TIC, AMC, CFZ, FEP, } \\
\text { CFP, CTX, FOX, CXM, CEF, SUL, } \\
\text { ATM }\end{array}$ \\
\hline EC59 & Urine & $i n t I 1+i n t I 2$ & $3.0+2.2$ & $\begin{array}{l}\operatorname{aacA4-aacCl-} \\
\operatorname{orfX-orfY-} \\
\operatorname{aadA1}+d f r A 1- \\
\text { sat1-aadA1 }\end{array}$ & $\begin{array}{l}b^{b l a_{\mathrm{CTX}-\mathrm{M}-3}}, \\
\text { bla }_{\mathrm{CMY}-15}\end{array}$ & $\begin{array}{l}\text { AMK, GEN, KAN, NET, STR, TOB, } \\
\text { AMP, PIP, TZP, TIC, AMC, CFZ, FEP, } \\
\text { CFP, CTX, FOX, CAZ, CXM, CEF, CIP, } \\
\text { NOR, SUL, TMP, SXT, TET, ATM, } \\
\text { CHL, NIT }\end{array}$ \\
\hline
\end{tabular}


Table 1 continued

\begin{tabular}{|c|c|c|c|c|c|c|}
\hline Strain & Origin & Integrase & $\begin{array}{l}\text { Integron } \\
\text { variable } \\
\text { region size } \\
\text { (kbp) }\end{array}$ & $\begin{array}{l}\text { Integron's } \\
\text { gene } \\
\text { cassettes }\end{array}$ & bla genes & Resistance pattern \\
\hline EC60 & Urine & intII & n.d. & n.d. & $b l a_{\mathrm{CTX}-\mathrm{M}-3}$ & $\begin{array}{l}\text { AMK, GEN, KAN, NET, STR, TOB, AMP, PIP, } \\
\text { TIC, AMC, CFZ, FEP, CFP, CTX, FOX, CXM, } \\
\text { CEF, NOR, SUL, TMP, SXT, TET, ATM }\end{array}$ \\
\hline EC61 & Urine & intII & 1.6 & dfrA1-aadA1 & $\begin{array}{l}\text { bla }_{\mathrm{CTX}-\mathrm{M}-55} \\
\text { bla }_{\mathrm{OXA}-1} \\
\text { bla }_{\mathrm{CMY}-15}\end{array}$ & $\begin{array}{l}\text { AMK, KAN, NET, STR, TOB, AMP, PIP, TIC, } \\
\text { AMC, CFZ, FEP, CFP, CTX, FOX, CXM, CEF, } \\
\text { CIP, NOR, SUL, TMP, SXT, TET, CHL }\end{array}$ \\
\hline EC62 & Urine & intII & 1.7 & $\begin{array}{r}\text { dfrA17- } \\
\text { aadA5 }\end{array}$ & bla $_{\mathrm{CTX}-\mathrm{M}-15}$ & $\begin{array}{l}\text { AMK, GEN, STR, AMP, PIP, TIC, AMC, CFZ, } \\
\text { FEP, CFP, CTX, FOX, CAZ, CXM, CEF, CIP, } \\
\text { NOR, SUL, ATM, NIT }\end{array}$ \\
\hline EC63 & Urine & - & - & - & $\begin{array}{c}\text { bla }_{\mathrm{CTX}-\mathrm{M}-15} \\
\text { bla }_{\mathrm{TEM}-1}\end{array}$ & $\begin{array}{l}\text { AMK, STR, TOB, AMP, PIP, TIC, CFZ, FEP, CFP, } \\
\text { CTX, FOX, CXM, CEF, CIP, NOR, SUL, TMP, } \\
\text { SXT, TET, ATM, CHL }\end{array}$ \\
\hline EC64 & $\begin{array}{c}\text { Vaginal } \\
\text { swab }\end{array}$ & intII & n.d. & n.d. & - & $\begin{array}{l}\text { AMK, GEN, KAN, NET, STR, TOB, AMP, PIP, } \\
\text { TIC, CFZ, FEP, CFP, CTX, FOX, CAZ, CXM, } \\
\text { CEF, CIP, NOR, SUL, TET, ATM }\end{array}$ \\
\hline EC65 & Urine & intII & 1.7 & $\begin{array}{r}d f r A 17- \\
\text { aadA5 }\end{array}$ & bla $_{\mathrm{CTX}-\mathrm{M}-15}$ & $\begin{array}{l}\text { KAN, STR, AMP, PIP, TIC, CFZ, FEP, CFP, CTX, } \\
\text { FOX, CXM, CEF, CIP, NOR, SUL, TMP, SXT, } \\
\text { TET, ATM }\end{array}$ \\
\hline EC66 & Urine & intII & 0.7 & $\begin{array}{l}\text { hypothetical } \\
\text { protein } \\
\text { gene }\end{array}$ & $\begin{array}{c}b^{b} a_{\mathrm{CTX}-\mathrm{M}-1} \\
b l a_{\mathrm{OXA}-1}\end{array}$ & $\begin{array}{l}\text { AMK, GEN, KAN, NET, STR, TOB, AMP, PIP, } \\
\text { TIC, AMC, CFZ, FEP, CFP, CTX, CAZ, CXM, } \\
\text { CEF, CIP, NOR, SUL, TMP, SXT, TET, ATM }\end{array}$ \\
\hline EC67 & Sputum & intII & 0.7 & $\begin{array}{l}\text { hypothetical } \\
\text { protein } \\
\text { gene }\end{array}$ & $\begin{array}{c}b^{b l a_{\mathrm{CTX}-\mathrm{M}-1}} \\
b l a_{\mathrm{OXA}-1}\end{array}$ & $\begin{array}{l}\text { AMK, GEN, KAN, NET, STR, TOB, AMP, PIP, } \\
\text { TIC, AMC, CFZ, FEP, CFP, CTX, CAZ, CXM, } \\
\text { CEF, CIP, NOR, SUL, TMP, SXT, TET, ATM, } \\
\text { NIT }\end{array}$ \\
\hline EC68 & Urine & intII & 0.7 & $\begin{array}{l}\text { hypothetical } \\
\text { protein } \\
\text { gene }\end{array}$ & $\begin{array}{c}b l a_{\mathrm{CTX}-\mathrm{M}-1} \\
b l a_{\mathrm{OXA}-1}\end{array}$ & $\begin{array}{l}\text { AMK, KAN, NET, STR, TOB, AMP, PIP, TIC, } \\
\text { AMC, CFZ, FEP, CFP, CTX, CAZ, CXM, CEF, } \\
\text { CIP, NOR, SUL, TMP, SXT, TET, ATM, NIT }\end{array}$ \\
\hline EC69 & Blood & intII & 0.7 & $\begin{array}{l}\text { hypothetical } \\
\text { protein } \\
\text { gene }\end{array}$ & $\begin{array}{c}b^{b l a_{\mathrm{CTX}-\mathrm{M}-1}}, \\
\text { bla }_{\mathrm{TEM}-1}\end{array}$ & $\begin{array}{l}\text { KAN, NET, STR, TOB, AMP, PIP, TIC, AMC, } \\
\text { CFZ, FEP, CFP, CTX, CAZ, CXM, CEF, CIP, } \\
\text { NOR, SUL, TMP, TET, ATM }\end{array}$ \\
\hline
\end{tabular}

$A M K$ Amikacin, $A M C$ amoxicillin-clavulanic acid, $A M P$ ampicillin, $A T M$ aztreonam, $C F Z$ cefazolin, $F E P$ cefepime, $C F P$ cefoperazone, $C T X$ cefotaxime, $F O X$ cefoxitin, $C A Z$ ceftazidime, $C X M$ cefuroxime, $C E F$ cephalothin, $C H L$ chloramphenicol, $C I P$ ciprofloxacin, GEN gentamicin, KAN kanamycin, NET netilmicin, NIT nitrofurantoin, NOR norfloxacin, PIP piperacillin, TZP piperacillin-tazobactam, STR streptomycin, SUL sulfamethoxazole, TET tetracycline, TIC ticarcillin, TOB tobramycin, TMP trimethoprim, $S X T$ trimethoprim-sulfamethoxazole

n.d. Not determined

a Class 2 integron

\section{Discussion}

Sixty-nine clinically relevant $E$. coli strains originating from extra-intestinal infections were comprised in the study. We analyzed the level of antimicrobial resistance, the presence of integrons and $\beta$-lactamases-coding genes. Comparison of two groups of strains: one isolated in 1999-2001 and another in 2008-2010 showed significant differences in drug resistance frequency, presence of integrons and $\beta$-lactamasecoding genes. The frequency of antimicrobial resistance to all antimicrobials beside imipenem, streptomycin, piperacillin/tazobactam, and sulfamethoxazole increased significantly, reaching high levels toward aminoglycosides, $\beta$-lactams and fluoroquinolones. It generally mirrors the trends in E. coli resistance, yet we must emphasize the fact that the frequency of resistance of strains isolated in 1999-2001 was already 
relatively high compared to other data. Turner (2008) has reported results of Meropenem Yearly Susceptibility Test Information Collection (MYSTIC) program from 2006 and its comparison with 2002. All antibiotics demonstrated reduction in activity against clinical isolates of E. coli in 2006 in comparison with the results from 2002: for gentamicin the percentage of sensitive strains was $91.7 \%$ (86.7\% in 2002), tobramycin $69.6 \% \quad(84.2 \%) \quad$ ciprofloxacin $73.3 \%$ $(82.1 \%)$, ceftazidime $86.6 \%(92.5 \%)$, piperacillin + tazobactam $85.9 \%$ (93.1\%), imipenem $99.7 \%$ (99.4\%) and amikacin $99 \%$ (not tested in 2002). According to data collected by the European Antimicrobial Resistance Surveillance System (EARSS and EARS-Net) regarding E. coli isolated from bloodstream infections, a significant increase of resistance in years 2002-2009 has been observed (Gagliotti et al. 2011). That regarded resistance to third-generation cephalosporins and combined resistance, i.e. resistance to two, three or four antimicrobials classes (aminoglycosides, aminopenicilins, third-generation cephalosporins and fluoroquinolones). We noted an increase in resistance to aminoglycosides, beta-lactams and fluoroquinolones. The EARSS survey from 2008 has reported the resistance against third-generation cephalosporines to be the most dynamic in Europe, which predicted increase in the number of ESBL-producing strains (European Centre for Disease Prevention and Control 2009). The EARS-Net report from 2010 has supported the remarkable Europe-wide decline of antimicrobial susceptibility in E. coli: in several countries both multidrug resistance and resistance frequency were increasing. The proportion of E. coli isolates resistant to third-generation cephalosporins increased significantly during 2006-2010 in half of the reporting countries. Among these isolates, a high proportion (65-100 \%) was identified as ESBL producers. These data indicate that ESBL production is highly prevalent in third-generation cephalosporinresistant E. coli in European hospitals (European Centre for Disease Prevention and Control 2011). In our research, the percentage of ESBL-positive strains increased significantly from 21.1 to $93.5 \%$ between 1999-2001 and 2008-2010. Most of the ESBLproducing isolates $(75.7 \%)$ had a $b l a_{\mathrm{CTX}-\mathrm{M}}$ gene: the frequency of strains with $b l a_{\mathrm{CTX}-\mathrm{M}}$ grew significantly in group 2 in comparison with group 1 . The analysis of the resistance frequency and the presence of bla $a_{\mathrm{CTX}-\mathrm{M}}$ genes reflected another tendency: the strains producing
CTX-M $\beta$-lactamases acquired resistance to other than $\beta$-lactams, classes of antimicrobials namely tetracycline $(P=0.002)$ and fluoroquinolones $(P<0.001)$. Surveys conducted worldwide have shown a growing resistance frequency to antimicrobials like tetracycline, gentamicin, tobramycin and ciprofloxacin in CTX-M producing E. coli (Pitout and Laupland 2008).

We also noticed the increase in the number of strains with integrons from 31.6 to $80.7 \%$. The presence of integrase genes was associated with increased frequency of resistance to each antimicrobial tested besides imipenem, piperacillin/tazobactam and ceftazidime $(P<0.05)$. The presence of integrons was also associated with multidrug resistance and the presence of ESBL phenotype and ESBL-encoding genes $(P<0.001)$. The genetic content of integrons comprised genes determining resistance toward aminoglycosides, sulfonamides and trimethoprim but the resistance of intI-positive isolates was far broader. In the first group of $E$. coli we detected three arrays of genes within integrons: dfrAl-aadA, dfrA17-aadA5, and $d f r A 12-o r f F-a a d A 2$. Gene cassette arrays like that are commonly appearing in E. coli isolates (Machado et al. 2005; El-Najjar et al. 2010). In the genomes of isolates from 2008 to 2010 we observed greater versatility of gene cassettes, more gene cassettes within an integron, appearance of class 2 integrons, and presence of more than one integron in bacterial genome. We detected six different integron-embedded gene cassette arrays: dfr2d, aadAl, dfrAl-aadA1, dfrA17aadA5, dfrA12-orfF-aadA2, and aacA4-aacC1-orfA$\operatorname{orf} B$-aadAl as well as cassettes coding for hypothetical proteins. All intIl-positive strains produced ESBL. The most often identified gene determining $\beta$-lactamase production was $b l a_{\mathrm{CTX}-\mathrm{M}}$ type, identified by sequencing as $b l a_{\mathrm{CTX}-\mathrm{M}-1}, b l a_{\mathrm{CTX}-\mathrm{M}-3}, b l a_{\mathrm{CTX}-\mathrm{M}-15}$, and $b l a_{\mathrm{CTX}-\mathrm{M}-55}$. In strains isolated from 1998 to 2001, only bla $_{\mathrm{CTX}-\mathrm{M}-3}$ was present. The CTX-M $\beta$-lactamases are now predominant in Poland and were noted for the first time in the late 1990s and identified as CTX-M-3. (Gniadkowski et al. 1998b; Livermore et al. 2007). In the following years, bla $a_{\mathrm{CTX}-\mathrm{M}-15}$ appeared possibly by point mutation in $b l a_{\mathrm{CTX}-\mathrm{M}-3}$ (Poirel et al. 2002). The CTX-M-15 lactamase is 100-fold more active against ceftazidime than CTXM-3 (Cartelle et al. 2004).

The genetic environment of bla $\mathrm{CTX}_{\mathrm{M}}$ genes in most of our isolates was homogenous and consisted of ISEcpl upstream $b l a_{\mathrm{CTX}-\mathrm{M}-1}, b l a_{\mathrm{CTX}-\mathrm{M}-3}$, and 
bla $a_{\mathrm{CTX}-\mathrm{M}-15}$. ISEcpl is frequently associated with $b l a_{\text {CTX-M }}$ genes and as it includes promoter sequences, it enhances otherwise poor $b l a_{\text {СтХ-M }}$ expression (Poirel et al. 2008). That element may also transpose downstream located fragments and thus facilitate the

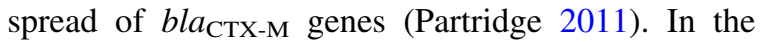
isolates from 2008 to 2010 beside integrons and $b l a_{\mathrm{CTX}-\mathrm{M}}$, there were also plasmid-mediated AmpC $\beta$-lactamases: $b l a_{\mathrm{CMY}-15}$ and $b l a_{\mathrm{DHA}}$. AmpC $\beta$-lactamases at high levels, hydrolyse penicillins, most cephalosporins, cephamycins and monobactams (Pitout 2012). In a survey comprising 13 Polish hospitals, bla $a_{\mathrm{CMY}}$ were identified in Proteus mirabilis only, and CMY-15 was the type of enzyme most common among them (Empel et al. 2008).

In summary, we found significant increase in resistance frequency including resistance to first line antibiotics like cephalosporins and fluoroquinolones, integron presence, and ESBL phenotype frequency. We also noticed significant increase in the frequency of bla $a_{\mathrm{CTX}-\mathrm{M}} \beta$-lactamases with appearance of bla $a_{\mathrm{CTX}-\mathrm{M}-15}$ variant and newer plasmid-encoded $\beta$-lactamases like CMY and DHA. We observed the emergence of strains with resistance to several classes of antimicrobials simultaneously with integrons, ESBL and AmpC $\beta$-lactamases coding genes. That may predict the spread of strains resistant to main classes of antimicrobials with no options for treatment apart from monobactams.

Open Access This article is distributed under the terms of the Creative Commons Attribution License which permits any use, distribution, and reproduction in any medium, provided the original author(s) and the source are credited.

\section{References}

Aragón ML, Mirelis B, Miró E, Gómez L, Rivera A, Coll P, Navarro F (2008) Increase in $\beta$-lactam-resistant Proteus mirabilis strains due to CTX-M- and CMY-type as well as new VEB- and inhibitor-resistant TEM-type $\beta$-lactamases. J Antimicrob Chemother 61:1029-1032

Cambray G, Guerout A, Mazel D (2010) Integrons. Annu Rev Genet 44:141-146

Cartelle M, del Mar TM, Molina F, Moure R, Villanueva R, Bou G (2004) High-level resistance to ceftazidime conferred by a novel enzyme, CTX-M-32, derived from CTX-M-1 through a single Asp240-Gly substitution. Antimicrob Agents Chemother 48:2308-2313

CLSI (2009) Performance standards for antimicrobial disk susceptibility tests; approved standard, 10th edn. M02A10. vol 29. No. 1
Daikos G, Kosmidis C, Tassios P, Petrikkos G, Vasilakopoulou A, Psychogiou M, Stefanou I, Avlami A, Katsilambros N (2007) Enterobacteriaceae bloodstream infections: presence of integrons, risk factors and outcome. Antimicrob Agents Chemother 51:2366-2372

De Champs C, Poirel L, Bonnet R, Sirot D, Chanal C, Sirot J (2002) Prospective survey of $\beta$-lactamases produced by ceftazidime-resistant Pseudomonas aeruginosa isolated in a French hospital in 2000. Antimicrob Agents Chemother 46:3031-3034

Dillon B, Thomas L, Mohmand G, Zelynsky A, Iredell J (2005) Multiplex PCR for screening of integrons in bacterial lysates. J Microbiol Methods 62:221-232

Eckert C, Gautier V, Arlet G (2006) DNA sequence analysis of the genetic environment of various bla $_{\mathrm{CTX}-\mathrm{M}}$ genes. J Antimicrob Chemother 57:14-23

El-Najjar NG, Farah MJ, Hashwa FA, Tokajian ST (2010) Antibiotic resistance patterns and sequencing of class I integron from uropathogenic Escherichia coli in Lebanon. Lett Appl Microbiol 51:456-461

Empel J, Baraniak A, Literacka E, Mrowka A, Fiett J, Sadowy E, Hryniewicz W, Gniadkowski M (2008) Molecular survey of $\beta$-lactamasesconferring resistance to newer $\beta$-lactams in Enterobacteriaceae isolates from Polish hospitals. Antimicrob Agents Chemother 52:2449-2454

European Centre for Disease Prevention and Control (2009) EARSS Annual Report 2008. Bilthoven

European Centre for Disease Prevention and Control (2011) Antimicrobial resistance surveillance in Europe 2010. Annual Report of the European Antimicrobial Resistance Surveillance Network (EARS-Net). Stockholm

Gagliotti C, Balode A, Baquero F, Degener J, Grundmann H, Gür D, Jarlier V, Kahlmeter G, Monen J, Monnet DL, Rossolini GM, Suetens C, Weist K, Heuer O, The EARS Net Participants (Disease Specific Contact Points for AMR) (2011) Escherichia coli and Staphylococcus aureus: bad news and good news from the European Antimicrobial Resistance Surveillance Network (EARS-Net, formerly EARSS), 2002 to 2009. Euro Surveill 16(11):pii=19819. Available online: http://www.eurosurveillance

Gniadkowski M, Schneider I, Jungwirth R, Hryniewicz W, Bauernfeind A (1998a) Ceftazidime-resistant Enterobacteriaceae isolates from three Polish hospitals: identification of three novel TEM- and SHV-5-type extendedspectrum $\beta$-lactamases. Antimicrob Agents Chemother 42:514-520

Gniadkowski M, Schneider I, Palucha A, Jungwirth R, Mikiewicz B, Bauernfeind A (1998b) Cefotaxime resistant Enterobacteriaceae isolates from a hospital in Warsaw, Poland: identification of a new CTX-M-3 cefotaximehydrolyzing $\beta$-lactamase that is closely related to the CTXM-1/MEN-1 enzyme. Antimicrob Agents Chemother 42:827-832

Hall RM, Collis CM (1995) Mobile gene cassettes and integrons: capture and spread of genes by site-specific recombination. Mol Microbiol 15:593-600

Kaper JB, Nataro JP, Mobley HL (2004) Pathogenic Escherichia coli. Nat Rev Microbiol 2:123-140

Leverstein-van Hall MA, Blok HEM, Rogier A, Donders T, Paauw A, Fluit AC, Verhoef J (2003) Multidrug resistance among Enterobacteriaceae is strongly associated with the 
presence of integrons and is independent of species or isolate origin. J Infect Dis 187:251-259

Lévesque C, Piché L, Larose C, Roy PH (1995) PCR mapping of integrons reveals several novel combinations of resistance genes. Antimicrob Agents Chemother 39:185-191

Li CR, Li Y, Zhang PA (2003) Dissemination and spread of CTX-M extended-spectrum $\beta$-lactamases among clinical isolates of Klebsiella pneumoniae in central China. Int J Antimicrob Agents 22:521-525

Li Y, Li Q, Du Y, Jiang X, Tang J, Wang J, Li G, Jiang Y (2008) Prevalence of plasmid-mediated AmpC $\beta$-lactamases in a Chinese university hospital from 2003 to 2005: first report of CMY-2-type AmpC $\beta$-lactamase resistance in China. J Clin Microbiol 46:1317-1321

Livermore DM, Canton R, Gniadkowski M, Nordmann P, Rossolini GM, Arlet G, Ayala J, Coque TM, Kern-Zdanowicz I, Luzzaro F, Poirel L, Woodford N (2007) CTXM: changing the face of ESBLs in Europe. J Antimicrob Chemother 59:165-174

Machado E, Cantón R, Baquero F, Galán JC, Rollán A, Peixe L, Coquel TM (2005) Integron content of extended-spectrum$\beta$-lactamase-producing Escherichia coli strains over 12 years in a single hospital in Madrid, Spain. Antimicrob Agents Chemother 49:1823-1829

Mazel D (2006) Integrons: agents of bacterial evolution. Nat Rev Microbiol 4:608-620

Mendonça N, Ferreira E, Louro D, ARSIP Participants, Caniça M (2008) Molecular epidemiology and antimicrobial susceptibility of extended- and broad-spectrum $\beta$-lactamaseproducing Klebsiella pneumoniae isolated in Portugal. Int $\mathrm{J}$ Antimicrob Agents 34:29-37

Mokracka J, Koczura R, Pawłowski K, Kaznowski A (2011) Resistance patterns and integron cassette arrays of Enterobacter cloacae complex strains of human origin. J Med Microbiol 60:737-743

Partridge SR (2011) Analysis of antibiotic resistance regions in Gram-negative bacteria. FEMS Microbiol Rev 35:820-855

Partridge SR, Tsafnat G, Coiera EJ, Iredell R (2009) Gene cassettes and cassette arrays inmobile resistance integrons. FEMS Microbiol Rev 33:757-784

Pitout JDD (2012) Extraintestinal pathogenic Escherichia coli: a combination of virulence with antibiotic resistance. Front Microbiol 3:9
Pitout JD, Laupland KB (2008) Extended-spectrum $\beta$-lactamase-producing Enterobacteriaceae: an emerging publichealth concern. Lancet Infect Dis 8:159-166

Poirel L, Gniadkowski M, Nordmann P (2002) Biochemical analysis of the ceftazidime-hydrolysing extended-spectrum b-lactamase CTX-M-15 and of its structurally related b-lactamase CTX-M-3. J Antimicrob Chemother 50:1031-1034

Poirel L, Naas T, Nordmann P (2008) Genetic support of extended-spectrum $\beta$-lactamases. Clin Microbiol Infect 14:75-81

Quiroga MP, Andres P, Petroni A, Soler Bistue AJC, Guerriero L, Vargas LJ, Zorreguieta A, Tokumoto M, Quiroga C, Tolmasky ME, Galas M, Centron D (2007) Complex class 1 integrons with diverse variable regions, including aac(6)$I b-c r$, and a novel allele, qnrB10, associated with ISCRI in clinical enterobacterial isolates from Argentina. Antimicrob Agents Chemother 51:4466-4470

Russo T, Johnson J (2000) A proposal for a new inclusive designation for extraintestinal pathogenic isolates of Escherichia coli: exPEC. J Infect Dis 181:1753-1754

Sáenz Y, Brinas L, Domínguez E, Ruiz J, Zarazaga M, Vila J (2004) Mechanisms of resistance in multiple-antibioticresistant Escherichia coli strains of human, animal, and food origins. Antimicrob Agents Chemother 48:3996-4001

Turner PJ (2008) Meropenem activity against European isolates: report on the MYSTIC (Meropenem Yearly Susceptibility Test Information Collection) 2006 results. Diagn Microbiol Infect Dis 60:185-192

Versalovic J, Koeuth T, Lupski JR (1991) Distribution of repetitive DNA sequences in eubacteria and application to fingerprinting of bacterial genomes. Nucleic Acids Res 19:6823-6831

Welch RA (2006) The genus Escherichia. In: Dworkin M, Falkow S, Rosenberg E, Schleifer KH, Stackebrandt E (eds) The prokaryotes 3rd edn, volume 6: Proteobacteria: gamma subclass. Springer, New York, pp 60-71

Weldhagen $\mathrm{G}$ (2004) Integron and $\beta$-lactamases: a novel perspective on resistance. Int J Antimicrob Agents 23:556-562

White PA, McIver CJ, Rawlinson WD (2001) Integrons and gene cassettes in the Enterobacteriaceae. Antimicrob Agents Chemother 45:2561-2658 